\title{
CONSTRUCTION OF A NEW FAMILY OF EFFICIENT IMBEDDED POLYNOMIALS WITH DISTINCT COEFFICIENTS
}

\section{Aftab Ahmad Malik and Nadeem Ahmad}

ABSTRACT: We propose a new family of multi-purpose imbedded polynomials having distinct coefficients. There exist relationships between various coefficients of the members of the family, which considerably reduce the computational cost of development as well as using any number of members of the family in a particular problem. Every member polynomial of degree $n$ going through $(n+1)$ focuses $\{(x i, y i): i=0,1 \ldots \ldots \ldots . n\}$ can be constructed very easily from another member having degree (n-1). In this paper, it is shown that the family of polynomials $\mathrm{M}$ exists and is efficient, reliable and more accurate as compared to other available techniques. The family has been successfully applied to the problem of interpolation in this paper. Therefore, the family $M$ is also called the Malik's Imbedded Interpolating Polynomials (M.I.I.P). The family $M$ gives similar results as compared to Lagrange Interpolation as for as accuracy is concerned but they are more efficient. The proposed polynomials are more efficient, more stable and more reliable as compared to other traditional interpolation methods due to remarkable reduction in mathematical operations. Our approach and the design of the method is different of available methods of prototype interpolation Methods. We have considered the drawbacks of other methods and eliminated from our approach. The superiority of the family is established and reported.

\section{INTRODUCTION}

The motive and incentive behind the development of the family $M$ is the idea of imbedded numerical multiterminal rules proposed by the present author in Malik[1],Malik[2], Genz and Malik[3]\& Genz and Malik[4], where computational cost in terms of mathematical operations of the higher degree rules is reduced by reusing a rule of lower degree.

While introducing the family $M$, we interject a polynomial $\mathrm{P}^{(\mathrm{n}-1)}(\mathrm{x})$ of degree less or equivalent to (n-1) passing through $n$ points $\left\{\left(\mathrm{x}_{i}, \mathrm{y}_{\mathrm{i}}\right): \mathrm{i}=0,1, \ldots \mathrm{n}-1\right\}$ and then add one point more point $\left(\mathrm{x}_{\mathrm{n}}, \mathrm{y}_{\mathrm{n}}\right)$ and interpolate a polynomial $\mathrm{P}^{(\mathrm{n})}(\mathrm{x})$ of degree less or equal to $\mathrm{n}$ using the incidents of $\mathrm{P}^{(\mathrm{n}-1)}(\mathrm{x})$. There exist useful and relations between the coefficients of $\mathrm{P}^{(\mathrm{n}-1)}(\mathrm{x})$ and $\mathrm{P}^{(\mathrm{n})}(\mathrm{x})$ which are developed in this paper.

We propose the Polynomials which shall be widely used in numerical Analysis, mathematics and Science in the form of equations to tackle several problems. It is well known that a polynomial is a relationship between known and unknown variables and constants. Nowadays, 
polynomials extensive used in curve and surface design in Computer graphics for modelling of the objects. Our polynomial shall cater for numerical description of the objects in terms of shape and size. Using these polynomials we may formulate points, line, polylines (a chain of connected line segments), polygons, smooth curves, wireframe models etc.

According to Griffiths \& Smith[6] in Lagrange interpolation high number of mathematical operations are required to compute an interpulating polynomials number of points $\left(x_{i}, y_{i}\right)$ increase. Moreover, there is exist another problem related to the efficiency of the Lagrangian approach if new data points are added to a set that has already been operated on, we shall have to use all points to interpolate the ploynomial afresh making the application inefficient. In the family $M$ both of these above mentioned problems have successfully overcome because we find the next degree polynomial by using the previously evaluated coefficient of the polynomial of the lower degree. Therefore, we overcome the difficulties mentioned Griffiths \& Smith
[6].

\section{Assumptions:}

All members of familyM allow:

- Some basic arithmetical and mathematical operations i.e. addition, subtraction, multiplications.

- Polynomials also obey commutative, associative, and distributive Laws.

- A sum or product of polynomials member is a polynomial.

- The derivative and anti-derivative of a member polynomial function is a polynomial function.

- The simple algebraic, trigonometric, polar, inverse trigonometric, hyperbolic, inverse hyperbolic, logarithmic and exponential function to be expressed in terms of transnational form \& in any appropriate member of the family $M$, using existing mathematical formalism and techniques.

- The degree $d$ of any member in nonnegative integer: $0<=\mathrm{d}<=$.

- Every member of M can be evaluated using Horner's Rule [5]. 


\section{Definitions and Notation:}

We define:

- Polynomial of degree 0 : $\quad \mathbf{P}^{(0)}(\mathbf{x})=B_{0}^{(0)}$ : A line parallel to $x$-axis

- Polvnomial of degree 1: $\quad \mathbf{P}^{(\mathbf{1})}(\mathbf{x})=B_{0}^{(1)}+B_{1}^{(1)} x$

- Polvnomial of degree 2:

$$
\mathrm{P}^{(2)}(\mathrm{x})=B_{0}^{(2)}+\mathrm{B}_{1}^{(2)} x+B_{2}^{(2)} \mathbf{x}^{2}
$$

- Polvnomial of degree $(n-1)$ passes through $n$ points $\left\{\left(x_{i}, y_{i}\right): i=0,1, \ldots(n-1)\right\}$

$$
P^{(n-1)}(x)=\sum_{i=0}^{i=n-1} B_{i}^{(n-1)} x^{i-1}
$$

- Polvnomial of degree $n$ goes through $(n+1)$ focuses $\{(x i, y i): i=0,1, \ldots n\}$

$$
P^{(n)}(x)=\sum_{i=0}^{i=n} B_{i}^{(n)} x^{i}
$$

- Basis Polynomial: We use Lagrange Interpolation Polynomial as basis polynomials to develop the family $M$, where

$$
\mathbf{M}=\left\{\mathbf{P}^{(\mathbf{0})}(\mathbf{x}), \mathbf{P}^{(\mathbf{1})}(\mathbf{x}), \mathbf{P}^{(2)}(\mathbf{x}), \ldots, \mathbf{P}^{(\mathbf{n}-1)}(\mathbf{x}), \mathbf{P}^{(\mathbf{n})}(\mathbf{x}), \ldots\right\}
$$

In Lagrange Interpolation, we write $\mathrm{n}^{\text {th }}$ degree Polynomial as $P^{(n)}(x)=\sum_{i=0}^{i=n} L_{i}(x) y_{i}$

where $L_{i}(x)$ is a function of $x$ called Lagrange coefficient having a either value zero or one s.t. $L_{i}\left(x_{j}\right)=0$ for $i !=j$ and $L_{i}\left(x_{j}\right)=1$ for $i=j$. It is like Keroneker delta and

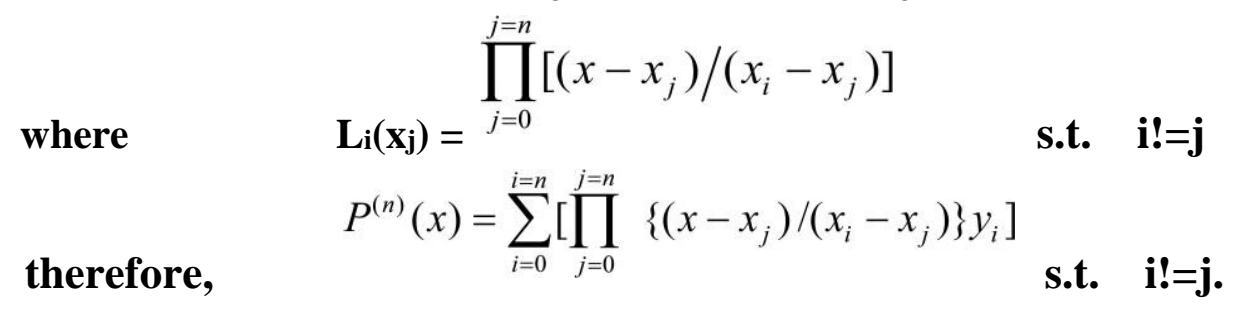

\section{Presentation and Construction of New Family M:}

$$
A_{i}^{(n)}=y_{i} \prod_{j=0}^{i=n}\left[1 /\left(x_{i}-x_{j}\right)\right]
$$

Assume:

$$
\text { s.t. } \quad \mathbf{i} !=\mathbf{j}
$$


Then

$$
P^{(n)}(x)=\sum_{i=0}^{i=n}\left[\left\{\prod_{j=0}^{j=n}\left(x-x_{j}\right)\right\} A_{i}\right]
$$

\section{s.t. $\quad \mathrm{i} !=\mathrm{j}$}

The product terms ${ }_{j=0}$ s.t $\mathbf{j} !=\mathbf{i}$ are very important for the construction of family

$$
\prod_{j=0}^{j=n}\left(x-x_{j}\right)
$$

$M$ of degree $n$. They are $n$ in number, excluding the one for $i=j$. Let us now compose our Polynomials:

Let us rewrite the polynomials as follows:

Polynomial of degree 1: $\mathbf{P}^{(\mathbf{1})}(\mathbf{x})=B_{0}^{(1)}+B_{1}^{(1)} x$

Using(10):

$$
\begin{aligned}
& \quad \text { where } B_{1}^{(1)}=A_{0}^{(1)}+A_{1}^{(1)}, B_{0}^{(1)}=-\left(A_{0}^{(1)} x_{1}+A_{1}^{(1)} x_{0}\right) \\
& A_{0}^{(1)}=y_{0} /\left(x_{0}-x_{1}\right)=y_{0} / \prod_{j=0 ; j !=0}^{j=1}\left(x_{0}-x_{j}\right)
\end{aligned}
$$

and

$$
A_{1}^{(1)}=y_{1} /\left(x_{1}-x_{0}\right)=y_{1} / \prod_{j=0, j !=1}^{j=1}\left(x_{1}-x_{j}\right)
$$

Polvnomial of degree 2: Now we Compute the coefficients of $\mathrm{P}^{(2)}(\mathrm{x})=B_{0}^{(2)}+\mathrm{B}_{1}^{(2)} x+B_{2}^{(2)}$ $\mathrm{x}^{2}$

as follows:

$$
B_{2}^{(2)}=A_{0}^{(2)}+A_{1}^{(2)}+A_{2}^{(2)}
$$

$B_{1}^{(2)}=-\left(B_{2}^{(2)}\left(x_{0}+x_{1}\right)-B_{1}^{(1)}\right)$

$B_{0}^{(2)}=\left(B_{2}^{(2)} x_{0} x_{1}+B_{1}^{(2)}\right)$

where

$$
\begin{aligned}
& \text { where } A_{0}^{(2)}=y_{0} /\left[\left(x_{0}-x_{1}\right)\left(x_{0}-x_{2}\right)\right]=y_{0} /\left[\prod_{j=0 ; j !=0}^{j=2}\left(x_{0}-x_{j}\right)\right] \\
& A_{1}^{(2)}=y_{1} /\left[\left(x_{1}-x_{0}\right)\left(x_{1}-x_{2}\right)\right]=y_{1} /\left[\prod_{j=0 ; j !=1}^{j=2}\left(x_{1}-x_{j}\right)\right]
\end{aligned}
$$$$
A_{2}^{(2)}=y_{2} /\left[\left(x_{2}-x_{0}\right)\left(x_{2}-x_{1}\right)\right]=y_{2} /\left[\prod_{j=0 ; j !=2}^{j=2}\left(x_{2}-x_{j}\right)\right]
$$

Notice in Equations(16) \& (17), we use the coefficients of polynomial of degree 1 to compute the coefficients of 2 nd degree.

Polvnomial of degree 3: This polynomial passes through 4- points $\left\{\left(x_{i}, y_{i}\right): i=0,1,2,3\right\}$. 
Actually working out and deriving the coefficients of $P^{(3)}(x)=B_{0}^{(3)}+B_{1}^{(3)} x+B_{2}^{(3)} x^{2}+B_{3}^{(3)} x^{3}$

it implies that : $\quad B_{3}^{(3)}=A_{0}^{(3)}+A_{1}^{(3)}+A_{2}^{(3)}+A_{3}^{(3)}$

$B_{2}^{(3)}=-\left(B_{3}^{(3)}\left(x_{0}+x_{1}+x_{2}\right)-B_{2}^{(2)}\right)$

$B_{1}^{(3)}=\left(B_{3}^{(3)}\left(x_{0} x_{1}+x_{0} x_{2}+x_{1} x_{2}\right)+B_{1}^{(2)}\right)$

$B_{0}^{(3)}=-\left(B_{3}^{(3)}\left(x_{0} x_{1} x_{2}\right)-B_{0}^{(2)}\right)$

where: $\quad A_{0}^{(3)}=y_{0} /\left[\left(x_{0}-x_{1}\right)\left(x_{0}-x_{2}\right)\left(x_{0}-x_{3}\right)\right]=y_{0} /\left[\prod_{j=0 ; j !=0}^{j=3}\left(x_{0}-x_{j}\right)\right]$

$A_{1}^{(3)}=y_{1} /\left[\left(x_{1}-x_{0}\right)\left(x_{1}-x_{2}\right)\left(x_{1}-x_{3}\right)\right]=y_{1} /\left[\prod_{j=0 ; j !=1}^{j=3}\left(x_{1}-x_{j}\right)\right]$

$A_{2}^{(3)}=y_{2} /\left[\left(x_{2}-x_{0}\right)\left(x_{2}-x_{1}\right)\left(x_{2}-x_{3}\right)\right]=y_{2} /\left[\prod_{j=0 ; j !=2}^{j=3}\left(x_{2}-x_{j}\right)\right]$

$A_{3}^{(3)}=y_{3} /\left[\left(x_{3}-x_{0}\right)\left(x_{3}-x_{1}\right)\left(x_{3}-x_{2}\right)\right]=y_{3} /\left[\prod_{j=0 ; j !=3}^{j=3}\left(x_{3}-x_{j}\right)\right]$

It may be noted that to compute the coefficients of a $3^{\text {rd }}$ Degree polynomial, we use four previously knownquantities without any fresh calculations i.e. $B_{2}^{(2)}$ $, B_{1}^{(2)}, B_{0}^{(2)} \& B_{3}^{(3)}$.

Table 1:

Number of

Subscripted Variables (x-coordinates)

\begin{tabular}{|c|c|c|c|c|}
\hline $\begin{array}{l}\text { Degr } \\
\text { ee }\end{array}$ & $\begin{array}{l}\text { Sum } \\
\text { with } \\
\text { Sing } \\
\text { le } \\
\text { ter } \\
\text { ms }\end{array}$ & $\begin{array}{l}\text { Sum } \\
\text { with } \\
\text { Pairs }\end{array}$ & $\begin{array}{l}\text { Tripl } \\
\text { ets }\end{array}$ & $\begin{array}{l}\text { Four } \\
\text { take } \\
\text { n at } \\
\text { a } \\
\text { time }\end{array}$ \\
\hline
\end{tabular}

We observe that there exist a peculiar pattern as for as the occurrence of $x$ coordinates such as $\left(\mathbf{x}_{0}, \mathbf{x}_{1}, \mathbf{x}_{2}, \mathbf{x}_{3}\right)$ in the coefficients of various Polynomials. They occur as sum of the products one taken at a time, sum of pairs, sum of triplet etc as shown below:

\begin{tabular}{|r|l|l|l|l|}
\hline $\mathbf{0}$ & $\begin{array}{l}\text { Non } \\
\text { e }\end{array}$ & & & \\
\hline$\bullet$ & $\mathbf{X} 0$ & & & \\
\hline$\bullet$ & $\begin{array}{l}\mathbf{X} 0+\mathbf{X} \\
1\end{array}$ & $\mathbf{X 0 X 1}$ & & \\
\hline$\bullet$ & $\mathbf{X} 0+$ & $\mathbf{X 0 X 1 +}$ & $\mathbf{X 0 X 1 X}$ & \\
$\mathbf{X} 1$ & $\mathbf{X 0 X 2 +}$ & $\mathbf{2}$ & \\
$+\mathbf{X} 2$ & $\mathbf{X} 1 \mathbf{X} 2$ & & \\
\hline$\bullet$ & $\mathbf{X} 0+$ & $\mathbf{X 0 X 1 + X 0}$ & $\mathbf{X 0 X 1 X}$ & $\mathbf{X 0 X 1}$ \\
\hline
\end{tabular}




\begin{tabular}{|l|l|l|l|l|}
\hline $\mathbf{X 1}$ & $\mathbf{X 2 +}$ & $2+$ & $\mathbf{X 2} \mathbf{X 3}$ \\
& $+\mathbf{X} 2+$ & $\mathbf{X 0 X 3 +}$ & $\mathbf{X 0 X 1 X}$ & \\
$\mathbf{X 3}$ & $\mathbf{X 1 X 2 +}$ & $\mathbf{3},+$ & \\
& & $\mathbf{X 1 X 3 +}$ & $\mathbf{X 0 X 2 X}$ & \\
& & $\mathbf{X 2 X 3}$ & $3+$ & \\
& & $\mathbf{X 1 X 2 X}$ & \\
& & & 3, & \\
\hline
\end{tabular}

In view of Table 1 and applying the strategy of Equations (12) to (29), we express our

Polynomial of Degree 4:

$P^{(4)}(x)=B_{0}^{(4)}+B_{1}^{(4)} x+B_{2}^{(4)} x^{2}+B_{3}^{(4)} x^{3}+B_{3}^{(4)} x^{4}$

Where

$B_{4}^{(4)}=A_{0}^{(4)}+A_{1}^{(4)}+A_{2}^{(4)}+A_{3}^{(4)}+A_{4}^{(4)}$

$$
\begin{aligned}
& B_{3}^{(4)}=-\left(B_{4}^{(4)}\left(x_{0}+x_{1}+x_{2}+x_{3}\right)-B_{3}^{(3)}\right) \\
& B_{2}^{(4)}=\left(B_{4}^{(4)}\left(x_{0} x_{1}+x_{0} x_{2}+x_{0} x_{3}+x_{1} x_{2}+x_{1} x_{3}+x_{2} x_{3}\right)+B_{2}^{(3)}\right) \\
& B_{1}^{(4)}=-\left(B_{4}^{(4)}\left(x_{0} x_{1} x_{2}+x_{0} x_{1} x_{3}+x_{0} x_{2} x_{3}+x_{1} x_{2} x_{3}\right)-B_{1}^{(3)}\right) \\
& B_{0}^{(4)}=-\left(B_{4}^{(4)}\left(x_{0} x_{1} x_{2} x_{3}\right)+B_{0}^{(3)}\right)
\end{aligned}
$$

In this rule, we use 5 coefficient values which are previously available i.e. $B_{4}^{(4)}, B_{3}^{(3)}, B_{2}^{(3)}, B_{1}^{(3)}$ and $B_{0}^{(3)}$. This aspect reduces the computational cost in terms of mathematical operations of our technique up to great extent, which will be analyzed in comparison with other existing methods. 


\section{REFERNCES:}

[1]. Malik A A [1977]: "Embedded Fully Symmetric Rules of of degree 5 and 7 for Multidimensional Integration"; M.Sc. Thesis University of Kent at Canterbury England. The work was supported by The British Council.

[2]. Malik A A [1980]: "Some new

Fully Symmetric Rules for multiple

Integrals with a Variable order Adaptive Algorithm"; Ph.D Thesis University of Kent at Canterbury

England. The work was supported by The British Council.

[3]. Genz AC and Malik A A [1980]: Algo :006: "An Adaptive Algorithm for Numerical Integration over an Ndimensional Rectangular Region Journal of Computational Applied Mathematics Volume 6 pp 295-302 Belgium.

[4]. GENZ, A. C., AND MALIK, A.A.[1983] : "An imbedded family of fully symmetric numerical integration rules. SIAM J. Numer. Anal. Volume 20 3, (June), PP580-588

[5]. Thomas H. Cormen, Charles E. Leiserson, Ronald L. Rivest, and Clifford
Stein. Introduction to Algorithms, Second Edition. MIT Press and McGraw-Hill, 2001. ISBN 0-262-03293-7. Problem 2-3 (pg. 39) and page 823 of section 30.1: Representation of polynomials.

[6]. Griffiths D. V., Smith I M. [1990]: "Numerical Methods For Engineers. by Chapman \& Hall. 